\title{
A load simulation method of piezoelectric actuator in FEM for smart structures
}

\author{
LI Min ${ }^{1}$, CHEN WeiMin ${ }^{2 \dagger}$, WANG MingChun ${ }^{1}$ \& JIA LiJie ${ }^{1}$ \\ ${ }^{1}$ School of Aeronautics Science and Engineering, Beihang University, Beijing 100191, China; \\ ${ }^{2}$ Division of Engineering Science Research, Institute of Mechanics, Chinese Academy of Sciences, Beijing 100190, China
}

More and more piezoelectric materials and structures have been used for structure control in aviation and aerospace industry. More efficient and convenient computation method for large complex structure with piezoelectric actuation devices is required. A load simulation method of piezoelectric actuation is presented in this paper. By this method, the freedom degree of finite element simulation is significantly reduced, the difficulty in defining in-plane voltage for multi-layers piezoelectric composite is overcome and the transfer computation between material main direction and the element main direction is simplified. The concept of simulation load is comprehensible and suitable for engineers of structure strength in shape and vibration control, thereby is valuable for promoting the application of piezoelectric material and structures in practical aviation and aerospace fields.

piezoelectric actuator, piezoelectric fiber composite, shape control, vibration control, drive characteristic

\section{Introduction}

In recent decades smart materials, such as piezoelectric actuator, have been currently used in structure control because of their high density of energy, wide range of response frequencies, good linear feature, flexible location and direct loading ${ }^{[1]}$. The researches performed in application of aviation and aerospace have been mainly focused on dynamic control and static control ${ }^{[2]}$ : (1) vibration and noise control, e.g. buffet control, flutter suppression to enlarge flight envelope, vibration control of engine and active noise control of aircraft; (2) shape control, e.g. shaping the wing of aircraft to improve aerodynamic performance, self-adapting micro-vehicle and control of vortex wake. And consequently large varieties of piezoelectric actuation devices and smart structures have been developed ${ }^{[3,4]}$. Among the broad kinds of smart materials and structures, the piezoelectric composite actuation devices ${ }^{[5-7]}$ benefiting from its higher power of actuating, directional nature of actuation, better flexibility and credibility, are becoming a basic control cell in practical aviation and aerospace fields. In fact with the improving of commercial performance piezo- electric fiber composite combined with carbon fiber composite has been applied to many projects ${ }^{[8-11]}$.

However the calculation technique and the corresponding computing code for smart materials and structures have not been developed deeply and fast as compared with the research of their application. As we know most of researchers employ programs written by themselves. As for commercial structure finite element codes, some have no piezoelectric element such as MSC/ NASTRAN currently used in the field of aviation and aerospace, some have $2 \mathrm{D}$ piezoelectric element that is too simplex to deal with complex structures such as piezoelectric fiber composite, and moreover, it needs too many elements of $3 \mathrm{D}$ elements to model a practical wing of aircraft. These computation disadvantages limit the application of smart materials and structures to engineering structures.

\footnotetext{
Received March 18, 2009; accepted April 29, 2009 doi: 10.1007/s11431-009-0211-4

Corresponding author (email: wmchen@imech.ac.cn)

Supported by the National Natural Science Foundation of China (Grant Nos. 10772183 and 10532070) and Foundation Program of Chinese Academy of Sciences (Grant No. KJCX2-YW-L07)
} 
In this paper a novel calculation method for piezoelectric structure, regarding piezoelectric actuator as a part of mechanical structure as common material and in addition simulating the actuation act by means of a group load, is presented. The significant advantage of this method is that it can be easily applied to current finite element code to deal with complex piezoelectric actuator. Furthermore, this method supports nonlinear calculation function and affords higher calculation efficiency since large numbers of fine 3D elements and freedom degrees are avoided.

Based on the governing equation of piezoelectric actuators, the load simulation method for piezoelectric actuating is deduced. And the discussion is focused on the piezoelectric sheet actuator used on the structure of wing. The method is valuable for promoting the application of piezoelectric material, especially piezoelectric fiber composite, in practical aviation and aerospace fields, because the concept of load simulation is comprehensible and the method is very suitable for structure strength engineers to research on shape control and vibration and acoustic noise reduction.

\section{Piezoelectric equations}

The mechanic-electric coupled constitutive relation includes linear elastic property and piezoelectric property. Based on the thermal-mechanical function and the energy rules the standard piezoelectric equation presented by IEEE Std - 1986 can be written as follows (in view of the application background, the symbols in the piezoelectric equations are suitable for mechanical workers):

$$
\begin{aligned}
\left\{\begin{array}{l}
D \\
\varepsilon
\end{array}\right\} & =\left[\begin{array}{ll}
\zeta^{\mathrm{T}} & d \\
d_{\mathrm{t}} & s^{\mathrm{E}}
\end{array}\right]\left\{\begin{array}{l}
E \\
\sigma
\end{array}\right\}, \\
\text { or }\left\{\begin{array}{l}
D \\
\sigma
\end{array}\right\} & =\left[\begin{array}{cc}
\zeta^{\mathrm{S}} & e \\
-e_{\mathrm{t}} & c^{\mathrm{E}}
\end{array}\right]\left\{\begin{array}{l}
E \\
\varepsilon
\end{array}\right\},
\end{aligned}
$$

where $D, E, \sigma$ and $\varepsilon$ are electric displacement $\left(\mathrm{C} / \mathrm{m}^{2}\right)$, electric field $(\mathrm{V} / \mathrm{m})$, stress $\left(\mathrm{N} / \mathrm{m}^{2}\right)$ and strain respectively; $\zeta^{\mathrm{T}}$ and $\zeta^{\mathrm{S}}$ are dielectric permittivity $(\mathrm{F} / \mathrm{m})$ at constant stress and constant strain respectively; $s$ and $c$ indicate flexibility $\left(\mathrm{m}^{2} / \mathrm{N}\right)$ and stiffness $\left(\mathrm{N} / \mathrm{m}^{2}\right) . d$ is piezoelectric strain coefficient $(\mathrm{m} / \mathrm{V})$ and $e$ is piezoelectric stress coefficient $\left(\mathrm{C} / \mathrm{m}^{2}\right)$. Superscript $\mathrm{T}, \mathrm{S}$, and $\mathrm{E}$ indicate the conditions of constant stress, constant strain and constant electric field respectively. Subscript $t$ indicates the transfer of matrix. Eqs. (1a) and (1b) are often called $d$-type piezoelectric equation and $e$-type piezoelectric equation respectively. The relation between $d$ and $e$ can be yielded from eq. (1) as follows:

$$
d=e s^{\mathrm{E}} \quad \text { or } \quad e=d c^{\mathrm{E}}
$$

and the relation between $\zeta^{\mathrm{T}}$ and $\zeta^{\mathrm{S}}$ is as follows:

$$
\zeta^{\mathrm{T}}-\zeta^{\mathrm{S}}=d e_{t}=e d_{t} .
$$

It should be noted that here $\varepsilon$ means total strain and $\sigma$ means mechanical stress (or elastic stress).

\section{Load simulation method of piezoelec- tric actuation}

Based on eq. (1) and the Hamilton Theorem, including grid displacement $U$ and grid electric potential $V$ as additional freedom degrees, we write element stiffness matrix $K_{11}^{\text {ele }}$, electromechanical coupling matrix $K_{12}^{\text {ele }}$ and permittivity matrix $K_{22}$ of piezoelectric element as follows:

$$
\begin{aligned}
K_{11}^{\text {ele }} & =\iiint_{v^{\text {ele }}} B_{t} c^{\text {ele }} c^{\mathrm{E}} B^{\text {ele }} \mathrm{d} v, \\
K_{12}^{\text {ele }} & =\iiint_{v^{e l e}} B_{t}^{\text {ele }} e_{t} B_{\varphi}^{\text {ele }} d v, \\
K_{22}^{\text {ele }} & =\iiint_{v^{\text {ele }}} B_{\varphi t}^{\text {ele }} \zeta^{S} B_{\varphi}^{\text {ele }} \mathrm{d} v,
\end{aligned}
$$

where $B^{\text {ele }}=A N^{\text {ele }}$ is the derivative matrix of element displacement interpolating matrix, $N^{\text {ele }}$ is the interpolating function of element displacement. $B_{\varphi}^{\text {ele }}=L N_{\varphi}^{\text {ele }}$ is the derivative matrix of element electric potential interpolating matrix, $N_{\varphi}^{\text {ele }}$ is the interpolating function of element electric potential. The strain-displacement relationship matrix $A$ is defined as $\varepsilon=A U$. The electric field and electric potential relationship matrix $L$ is defined as $E=L V$. Then the governing equations of piezoelectric structure are written as

$$
\begin{gathered}
{\left[K_{11}\right] U-\left[K_{12}\right] V+[M] \ddot{U}=F,} \\
{\left[K_{12}\right]_{t} U+\left[K_{22}\right] V=Q,}
\end{gathered}
$$

where $M$ is mass matrix, $F$ is external load vector including surface force and volume force, and $Q$ is free charge vector including surface charge and volume charge. To an actuator, the external grid voltage of piezoelectric element is applied with a fixed value by external action (e.g. the power amplifier), that can be regarded as a constraint boundary condition. When the distance between two electrodes is much smaller than 
the size of electrode surface, the electric field may be regarded as constant. Meanwhile the influence of changing internal grid voltage on the value of total electric field belongs to a neglectable higher order effect. Furthermore in finite element simulation the piezoelectric part between two electrodes is often meshed into elements with only one layer, so the grid voltage is set to be a given value. Now comparing to the traditional mechanical equation we move the additional term $K_{12} V$, indicating the coupling between the mechanism and the electrics, to the right side of eq. (5a) so that the action of piezoelectric actuation may be regarded as an equivalent load $F_{\mathrm{EQ}}$. And the element equivalent load $F_{\mathrm{EQ}}^{\text {ele }}$ is written as

$$
\begin{aligned}
& F_{\mathrm{EQ}}^{\mathrm{ele}}=\iiint_{v^{\mathrm{ele}}} B_{t}^{\mathrm{ele}} e_{t} B_{\varphi}^{\mathrm{ele}} \mathrm{d} v \cdot V \\
& =\iiint_{v^{\mathrm{ele}}} B_{t}^{\mathrm{ele}} e_{t} E \mathrm{~d} v=\iiint_{v^{\mathrm{ele}}} B_{t}^{\text {ele }} c^{\mathrm{E}} \varepsilon^{\mathrm{E}} \mathrm{d} v .
\end{aligned}
$$

And the element equivalent elastic force is

$$
K_{11}^{\text {ele }} U=\iiint_{v^{\text {ele }}} B_{t}^{\text {ele }} c^{\mathrm{E}} \varepsilon^{\mathrm{e}} \mathrm{d} v .
$$

Comparing eqs. (6) and (7) it can be seen that the equivalent piezoelectric stress is $c^{\mathrm{E}} \varepsilon^{\mathrm{E}}$, or the equivalent piezoelectric load should produce an elastic strain having the same value with the piezoelectric strain.

An alternative and simpler method decomposing the strain into two parts will also present the piezoelectric actuation equation so as to obtain a more directive expression for actuation load. The total strain

$$
\varepsilon=\varepsilon^{\mathrm{e}}+\varepsilon^{\mathrm{E}},
$$

where $\varepsilon^{\mathrm{e}}$ and $\varepsilon^{\mathrm{E}}$ are elastic strain and piezoelectric strain respectively.

$$
\begin{aligned}
\because \quad & \varepsilon^{\mathrm{e}}=s^{\mathrm{E}} \sigma, \quad \varepsilon^{\mathrm{E}}=d_{t} E, \\
\therefore \quad & \varepsilon=s^{\mathrm{E}} \sigma+d_{t} E \\
\text { or } \quad & \sigma=c^{\mathrm{E}}\left(\varepsilon-\varepsilon^{\mathrm{E}}\right)=c^{\mathrm{E}} \varepsilon-e_{t} E .
\end{aligned}
$$

Eqs. (10a) and (10b) express the total strain in eq. (1a) and the elastic stress in eq. (1b) respectively. It is found in eqs. (8) and (9) that the difference of piezoelectric material from the ordinary mechanical material is laid on the additional term, $\varepsilon^{\mathrm{E}}$, which is deduced from the simulating load of actuator:

$$
F_{i}=\int_{A_{i}} c^{\mathrm{E}} \varepsilon^{\mathrm{E}} \mathrm{d} A=\int_{A_{i}} c^{\mathrm{E}} d_{t} E \mathrm{~d} A,
$$

where $A_{i}$ is the area of piezoelectric element. By eq. (11) we may simulate the actuation by means of loading a force at the grid or on the surface of the piezoelectric element.

If a simulation force that can produce piezoelectric strain $\varepsilon^{\mathrm{E}}$ is used, then only a pure mechanical model including the mechanical property of piezoelectric material in the structure model needs to be considered. That is to say, by means of the simulative load of piezoelectric actuation and ordinary finite element code we can implement numerical simulation of design and analysis for smart material structures. In the following section we will give various expressions of simulation loads for piezoelectric actuation devices such as piezoelectric bar, piezoelectric sheet and piezoelectric fiber composite that are often used in practical smart materials and structures.

\section{Load expressions for various piezoelectric actuation devices}

If only the piezoelectric actuator is considered, the piezoelectric strains and deformations in three directions produced by the simulation loads from eq. (11) are respectively consistent with the values computed by numerical simulation using piezoelectric element. It should be pointed out that a free piezoelectric strain is not capable of producing any actuation effect. So the criterion of load simulation would not be considered for case of free piezoelectric strain.

The further simplified load expressions for various actuation devices will be presented below, which are deduced under the constrain condition of an expectant piezoelectric strain and consequently an expectant structure deformation should be produced under the simulation load.

\subsection{Actuation model for piezoelectric bar using function of $\boldsymbol{d}_{33}$}

A piezoelectric bar that connects with the host structure in direction 3 (shown in Figure 1) is polarized in direction 3 and is applied to electric filed only in this direction, i.e. only $E_{3} \neq 0$. When the piezoelectric bar performs as an axial actuator, only the piezoelectric strain in direction 3 produces an actuation effect on the host structure, meanwhile the free strain in directions 1 and 2 will not produce actuation effect. Thus the actuation loads in directions 1 and 2 are equal to zero, or $F_{1}=F_{2}=0$, and the actuation load in direction 3 is simply given by the following formula:

$$
F_{3}=E_{\mathrm{PZT}}^{3} A_{3} d_{33} E_{3},
$$




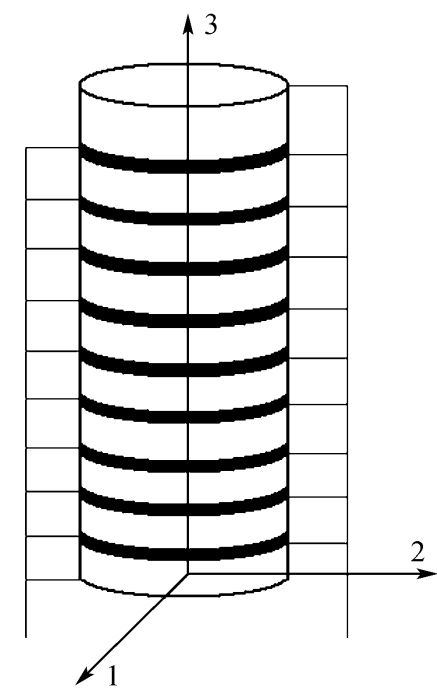

Figure 1 Sketch of a piezoelectric bar.

where $E_{\mathrm{PZT}}^{3}$ and $A_{3}$ are the Young's modulus and the section area normal to direction 3 respectively. Although the strain of piezoelectric bar in direction 1 (or 2) due to the Poisson's effect has a different value with the true piezoelectric strain in direction 1 (or 2), it does not influence the actuation and structure deformation control in direction 3 .

The main advantage of this simulation method is that in finite element model only 1D bar element is enough to simulate the piezoelectric actuation structure and the freedom degree is significantly decreased by avoiding use of 3D piezoelectric element.

\subsection{Actuation model for piezoelectric sheet using function of $d_{31}$ (or $\left.d_{32}\right)$}

The piezoelectric ceramic actuator shown in Figure 2 is often used to control the shape or vibration for thin plate in which the piezoelectric sheets (e.g. PZT5H) are bonded on the top and bottom surfaces of the host structure and an actuation moment is produced by means of applying a couple of opposite voltages on two piezoelectric sheets. The piezoelectric sheet is polarized in direction 3 and is applied to an electric filed only in this direction, i.e. $E_{3} \neq 0$. It is capable of affording actuation strains in directions 1 and 2 (free strain in direction 3 not affording actuation action). Generally, the thickness $t$ of thin plate is much smaller than the length $l$ or the width $w$ of plate, so it is a plane stress problem, or $\sigma_{3}=0$. The elastic constitutive equation for piezoelectric sheet with orthotropic property is written as

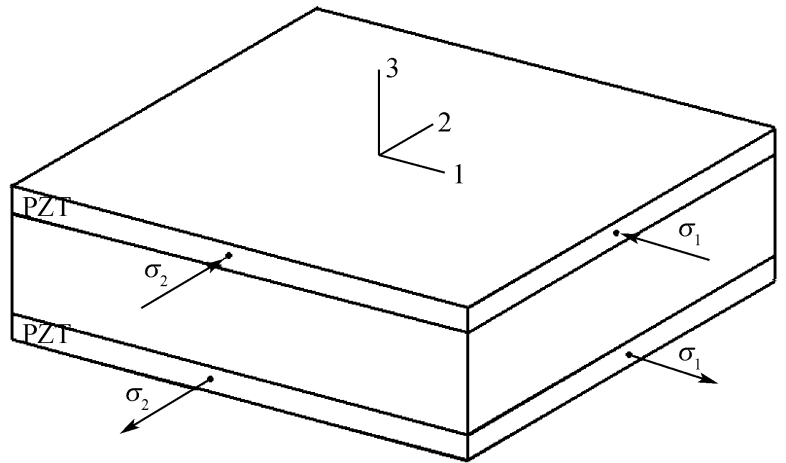

Figure 2 Piezoelectric sheets are bonded on the top and bottom surfaces of the host structure.

$$
\begin{gathered}
{\left[\begin{array}{c}
\sigma_{1} \\
\sigma_{2} \\
\sigma_{3} \\
\sigma_{4} \\
\sigma_{5} \\
\sigma_{6}
\end{array}\right]=\left[\begin{array}{cccccc}
c_{11} & c_{12} & c_{13} & 0 & 0 & 0 \\
c_{21} & c_{22} & c_{23} & 0 & 0 & 0 \\
c_{31} & c_{32} & c_{33} & 0 & 0 & 0 \\
0 & 0 & 0 & c_{44} & 0 & 0 \\
0 & 0 & 0 & 0 & c_{55} & 0 \\
0 & 0 & 0 & 0 & 0 & c_{66}
\end{array}\right]\left[\begin{array}{c}
\varepsilon_{1} \\
\varepsilon_{2} \\
\varepsilon_{3} \\
\varepsilon_{4} \\
\varepsilon_{5} \\
\varepsilon_{6}
\end{array}\right] .} \\
\because \quad \sigma_{3}=c_{31} \varepsilon_{1}+c_{32} \varepsilon_{2}+c_{33} \varepsilon_{3}=0, \\
\therefore \quad \varepsilon_{3}=-\frac{c_{31} \varepsilon_{1}+c_{32} \varepsilon_{2}}{c_{33}}, \\
=\frac{c_{11} c_{33}-c_{13} c_{31}}{c_{33}} \varepsilon_{1}+\frac{c_{12} c_{33}-c_{13} c_{32}}{c_{33}} \varepsilon_{2} .
\end{gathered}
$$

It is seen that the elastic matrix is symmetric and in 1-2 plane the piezoelectric plate is isotropic.

$$
\begin{aligned}
& \therefore \quad c_{13}=c_{31}, c_{23}=c_{32}, \quad c_{13}=c_{23}, \\
& \sigma_{1}=\frac{c_{11} c_{33}-c_{13}^{2}}{c_{33}} \varepsilon_{1}+\frac{c_{12} c_{33}-c_{13}^{2}}{c_{33}} \varepsilon_{2} .
\end{aligned}
$$

The strain produced by the equivalent piezoelectric load is piezoelectric strain written as

$$
\begin{gathered}
\varepsilon^{\mathrm{E}}=\mathrm{d}_{t} E=\left[\begin{array}{ccc}
0 & 0 & d_{31} \\
0 & 0 & d_{32} \\
0 & 0 & d_{33} \\
0 & d_{24} & 0 \\
d_{15} & 0 & 0 \\
0 & 0 & 0
\end{array}\right]\left[\begin{array}{c}
E_{1} \\
E_{2} \\
E_{3}
\end{array}\right]=\left[\begin{array}{c}
d_{31} E_{3} \\
d_{32} E_{3} \\
d_{33} E_{3} \\
d_{24} E_{2} \\
d_{15} E_{1} \\
0
\end{array}\right] . \\
\because E_{1}=E_{2}=0, E_{3} \neq 0, \text { and } d_{31}=d_{32}, \\
\therefore \sigma_{1}=\frac{d_{31} E_{3}}{c_{33}}\left[\left(c_{11}+c_{12}\right) c_{33}-2 c_{13}^{2}\right] .
\end{gathered}
$$


Similarly we have $\sigma_{2}=\sigma_{1}$.

The equivalent piezoelectric load is assumed to act on the center line of the thickness of the piezoelectric sheet. Then the produced bending moment (shown in Figure 3) by a couple of opposite voltages applied on two piezoelectric sheets are written respectively as

$$
\begin{gathered}
M_{\mathrm{W}}=\sigma_{1} w t_{\mathrm{p}}\left(t_{\mathrm{p}}+t\right), \\
M_{\mathrm{T}}=\sigma_{2} l t_{\mathrm{p}}\left(t_{\mathrm{p}}+t\right),
\end{gathered}
$$

where $t$ and $t_{\mathrm{p}}$ are the thickness of the thin plate and piezoelectric sheet respectively.

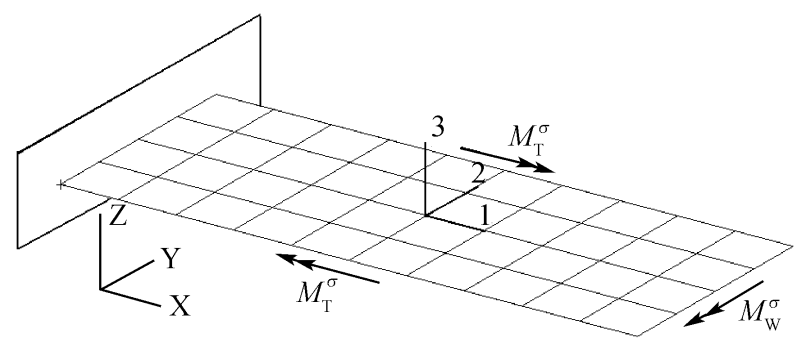

Figure 3 The moments produced by PZT and its distribution.

From Figure 3 an interesting phenomenon is found that the moments of the adjacent internal elements along the same boundary counteract each other. So the final simulation load acted only on the out boundary of the piezoelectric structure. Meanwhile the freedom degree is significantly decreased by avoiding large number of 3D piezoelectric elements. The strain in direction 3 of piezoelectric actuator computed by eq. (14) is not $d_{33} E_{3}$, but it could be ignored for a plane stress problem discussed here.

\subsection{Actuation model for piezoelectric fiber compos- ite using function of $d_{11}$ (or $\left.d_{12}\right)$}

The piezoelectric actuation structure shown in Figure 4

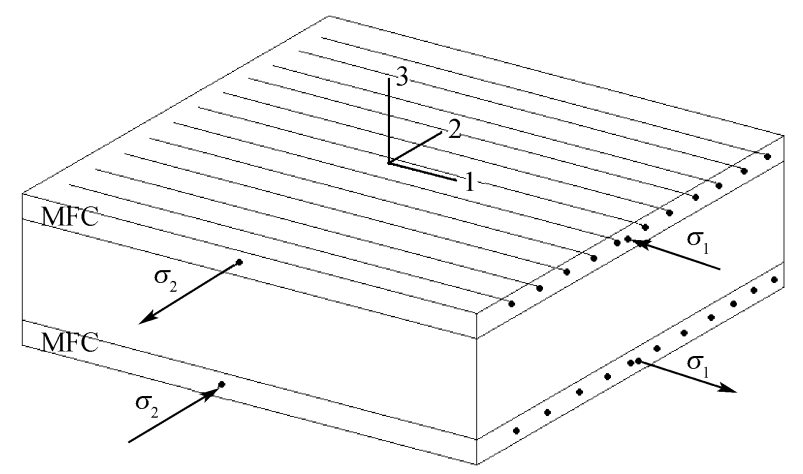

Figure 4 Two piezoelectric fiber-composite sheets are bonded on the host structure. has been often used to control the shape or vibration for thin plate in which piezoelectric fiber-composite sheets (e.g. Macro Fiber Composite called MFC) are bonded on the top and bottom surfaces of the host structure and a couple of actuation bending moments are developed by applying a couple of opposite voltages on the piezoelectric fibers.

Piezoelectric fiber is polarized in direction 1 and the electric field in this direction $E_{1} \neq 0$. The actuator is capable of producing actuation strain in directions 1 and 2 (free strain in direction 3 not performing actuation action), and it is approximately isotropic in plane 2-3.

Similar to the problem discussed in section 3.2 we also have $\sigma_{3}=0$ except the piezoelectric strain being

$$
\begin{gathered}
\varepsilon^{\mathrm{E}}=\mathrm{d}_{t} E=\left[\begin{array}{ccc}
d_{11} & 0 & 0 \\
d_{12} & 0 & 0 \\
d_{13} & 0 & 0 \\
0 & 0 & 0 \\
0 & 0 & 0 \\
0 & 0 & 0
\end{array}\right]\left[\begin{array}{c}
E_{1} \\
E_{2} \\
E_{3}
\end{array}\right]=\left[\begin{array}{c}
d_{11} E_{1} \\
d_{12} E_{1} \\
d_{13} E_{1} \\
0 \\
0 \\
0
\end{array}\right], \\
\sigma_{1}=\frac{c_{11} c_{33}-c_{13} c_{31}}{c_{33}} d_{11} E_{1}+\frac{c_{12} c_{33}-c_{13} c_{32}}{c_{33}} d_{12} E_{1}, \\
\sigma_{2}=\frac{c_{21} c_{33}-c_{23} c_{31}}{c_{33}} d_{11} E_{1}+\frac{c_{22} c_{33}-c_{23} c_{32}}{c_{33}} d_{12} E_{1} .
\end{gathered}
$$

Combining with eq. (19) the moment is obtained.

Another point that should be noted is that here we have $\sigma_{1} \neq \sigma_{2}$ but in section $4.2 \sigma_{1}=\sigma_{2}$. In fact $\sigma_{1}$ often has different signal from $\sigma_{2}$. And that brings an interesting consequence.

Although both cases of piezoelectric sheet in section 4.2 and piezoelectric fiber-composite sheet in section 4.3 belong to plane stress problem, the radius of the stress circle for the latter one has a maximum value of shear stress $\tau_{\max }=\left(\sigma_{1}-\sigma_{2}\right) / 2$, whereas the stress circle for the former one degenerates into one point $\mathrm{A}$ as shown in Figure 5. That means in the actuation plane $O X Y$ in structure coordinate the shear stress of piezoelectric material is always zero or the moment is produced only by normal stress. But for piezoelectric fiber-composite sheet, the moments are produced by the normal stress and the shear stress as shown in Figure 6. Especially, the moment by shear stress on different boundaries has significant influence on the structure torsion deformation, e.g. in Figure 7 moments $M_{\mathrm{T}}^{\tau}$ and $M_{\mathrm{W}}^{\tau}$ produced by 

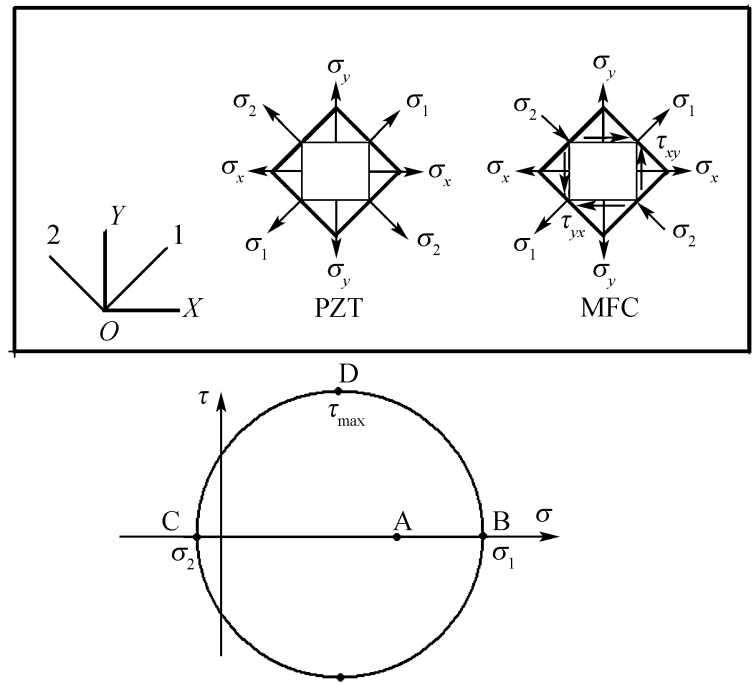

Figure 5 Stress distribution of piezoelectric material on actuation plane in structure coordinate.

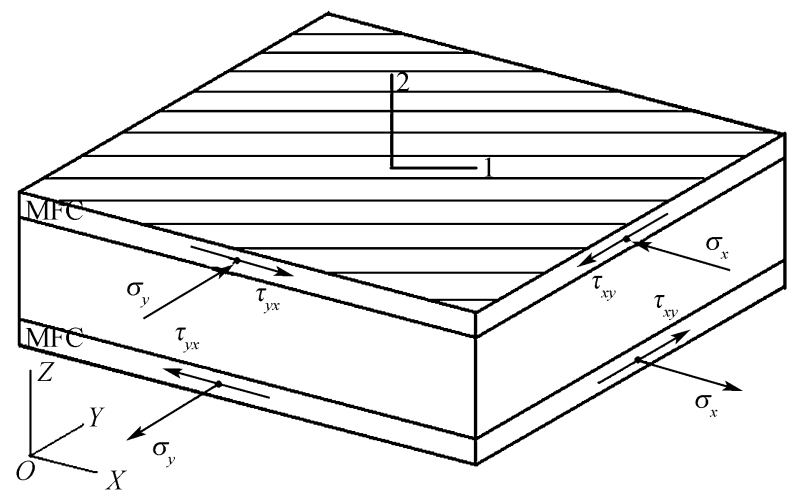

Figure 6 Stress distribution of piezoelectric element for MFC in structure coordinate.

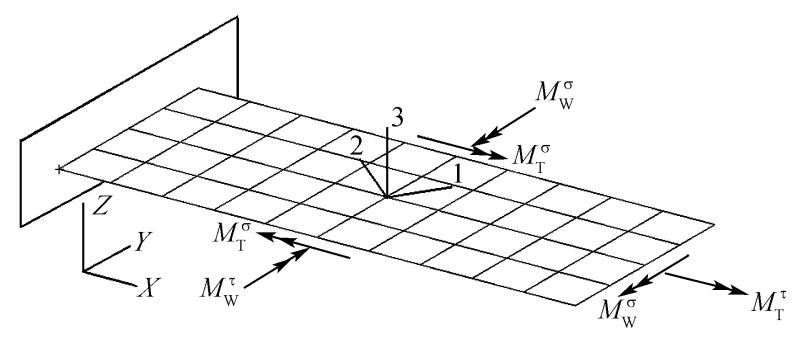

Figure 7 The moments produced by MFC in structure coordinate.

shear stress have torsion effect on the structure. This is much different from case of piezoelectric ceramic sheet where the effects of torsion produced by normal stress usually counteract each other.

Generally speaking, by using piezoelectric fiber composite and selecting appropriate layer angle one can control the structure shape, e.g. bending or twisting a plate, more accurately and effectively. The torsion effect is especially important to the shape control of aircraft wing where the change of the attack angle mainly developed by the torsion effect is the key for shaping wing to improve the air force distribution on the wing.

\section{Numerical examples}

The simulation load will be examined on the plate models for piezoelectric sheet and piezoelectric fiber-composite sheet excluding the simpler one, piezoelectric bar model, which can be directly validated by analytical deduction.

\subsection{Piezoelectric ceramic sheet actuation model}

The piezoelectric sheet is made from PZT5H with: stiffness coefficients $\left(10^{10} \mathrm{~N} / \mathrm{m}^{2}\right), \quad c_{11}^{\mathrm{E}}=12.6, \quad c_{12}^{\mathrm{E}}=7.95$, $c_{13}^{\mathrm{E}}=8.41, c_{33}^{\mathrm{E}}=11.7, c_{44}^{\mathrm{E}}=2.35, \quad c_{55}^{\mathrm{E}}=c_{66}^{\mathrm{E}}=2.3 \quad$ (both the stress components $\sigma_{i}$ and strain components $\varepsilon_{i}$ arrange in order of $11,22,33,12,23,31)$. Relative permittivity $\varepsilon_{11}^{\mathrm{S}} / \varepsilon_{0}=1700, \quad \varepsilon_{33}^{\mathrm{S}} / \varepsilon_{0}=1470$ and piezoelectric stress-coefficient $\left(\mathrm{C} / \mathrm{m}^{2}\right) e_{31}=-6.5, e_{23}=23.3, e_{24}=17.0$.

The plate model with geometry size $l \times w \times h=240$ $\mathrm{mm} \times 60 \mathrm{~mm} \times 3.6 \mathrm{~mm}^{3}$ is shown in Figure 8 where the end $(y=0)$ of plate is fixed. PZT5H wafers with thickness $0.3 \mathrm{~mm}$ are bonded on the top and bottom surfaces of an alumina plate with Young's modulus $E=70.3 \mathrm{GPa}$ and Poisson's ratio $\mu=0.345$.

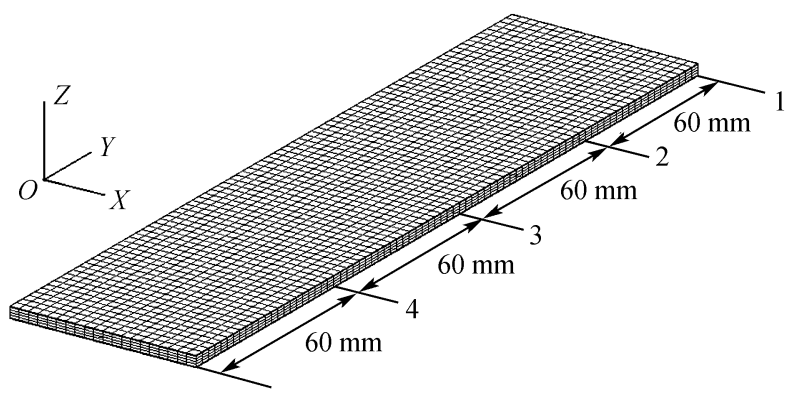

Figure 8 Finite element model.

Finite element simulation is executed on two models, one called Model A employs ANSYS code where 3D piezoelectric element (SOLID 5) is used, another one called Model B employs NASTAN code where ordinary mechanical plate element (2D composite plate element QUAD4) combining with simulation force loading according to eq. (19) is used. For the reason of result comparison, both models have the same element size $l \times w=3 \mathrm{~mm} \times 3 \mathrm{~mm}$ but different meshes through plate thickness. In Model A the thickness is meshed into 8 
layers, i.e. respectively, 2 layers with thickness $0.15 \mathrm{~mm}$ for the PZT5H wafer on the two sides of the surfaces and 4 layers with thickness $0.75 \mathrm{~mm}$ for the Al plate. In order to make the computation process convergent, in Model A 12800 elements and consequently 60000 freedom degrees (including electric potential degree) are employed, whereas in Model B element number is merely 1600 .

By comparing the deformation results of the two models induced by acting voltage on the piezoelectric layers, one would examine the simulation method presented in this paper. A voltage with value of $100 \mathrm{~V}$ is applied on the surfaces of PZ5H layer, and then the deformations of the cantilever plate are computed. In Model B a group of simulation forces, computed according to eq. (19), including a moment $0.459 \mathrm{Nm}$ equably distributed on out chord and a moment $\pm 1.836 \mathrm{Nm}$ equably distributed on leading and trailing edges are applied. Then the numerical results are plotted by means of displacements in the center line of plate middle plane along wingspan (shown in Figure 9) and displacements of plate middle plane at different chords (shown in Figure 10).

It shows that the relative error (based on the 3D model) of the maximum displacement of two results is less than $0.06 \%$. And in Figure 10 every chord almost keeps a horizontal line while small local attack angle only occurs at leading or trailing edge. So this kind of actuation device is suitable for bending control. If a torsion effect is required, the differential action of bending could be used to realize torsion but the effect is not very remarkable ${ }^{[12]}$.

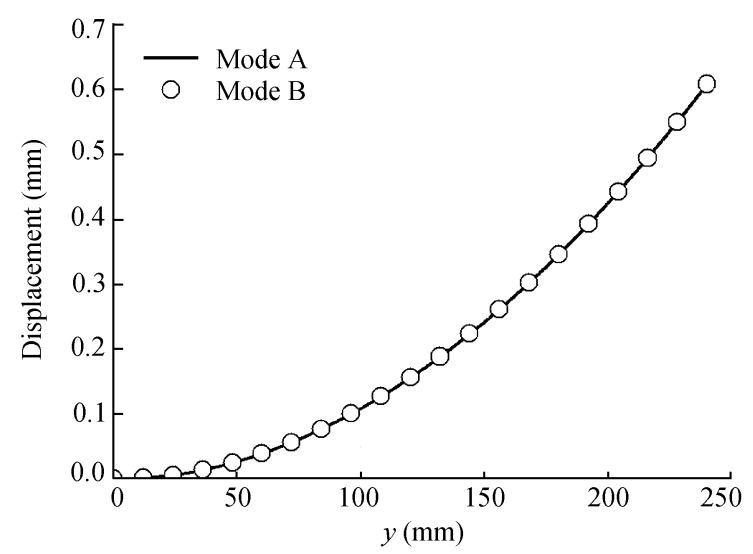

Figure 9 Displacement in the center line of plate middle plane along wingspan.

\subsection{Piezoelectric fiber-composite actuation model}

Here the piezoelectric actuator is MFC and the geometry model is the same as section 4.1, the mechanical properties of MFC defined in material main axis are ${ }^{[8]}$. $E_{1}=30.34 \mathrm{GPa}, E_{2}=15.86 \mathrm{GPa}, \mu_{12}=0.31, \mu_{21}=0.16$, $G_{12}=5.52 \mathrm{GPa}$, and with electric properties as piezoelectric strain coefficient $\left(\times 10^{-12} \mathrm{~m} / \mathrm{V}\right) d_{11}=400, d_{12}=-170$. It is assumed that the material is isotropic in 2-3 plane, $\mu_{23}=0.31$ and relative permittivity $\varepsilon_{11}^{\mathrm{S}} / \varepsilon_{0}=1700$, $\varepsilon_{33}^{\mathrm{S}} / \varepsilon_{0}=1470$. The electric field is $1000 \mathrm{~V} / \mathrm{mm}$.

Two layer schemes are examined. In the first scheme direction 1 of MFC is laid along $y$ direction of the global coordinate (Figure 8). The displacement results are shown in Figures 11 and 12 which show that the relative error of the maximum displacement of two models is less than $0.3 \%$. And one meaningful difference from the model with PZT5H actuator is the wave-like deformed shape of chordwise due to MFC being capable of producing a group of piezoelectric strain with opposite sign in directions 1 and 2 but PZT5H with the same sign.

In another scheme direction 1 of MFC is laid $+45^{\circ}$ from $y$ axis. In this scheme the material main direction is no longer consistent with the element main direction. In Model B the load is still conveniently applied by directly transferring the simulation stress from material main direction (directions 1 and 2$)$ to structure coordinate $(x$ axis and $y$ axis) (shown in Figure 5). But in Model A one must calculate the material mechanical and electrical properties defined in element coordinate, since the material coordinate could not be defined independently in

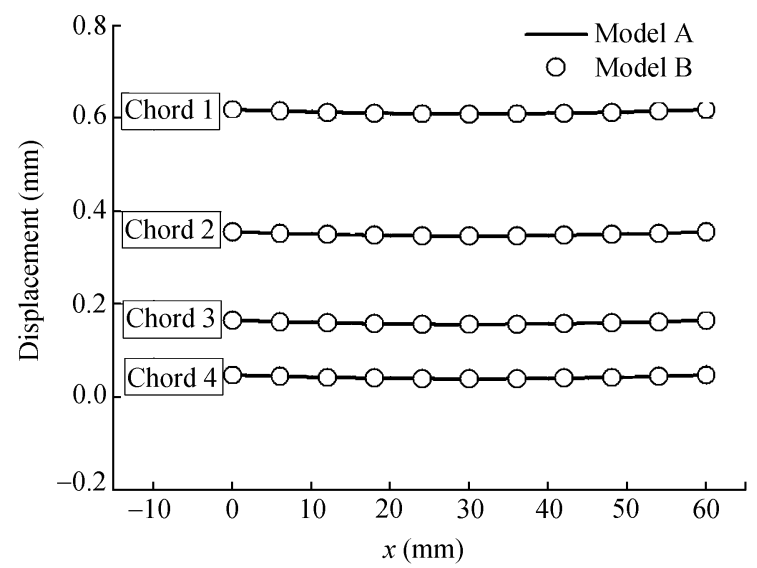

Figure 10 Displacements of plate middle plane at different chords. 


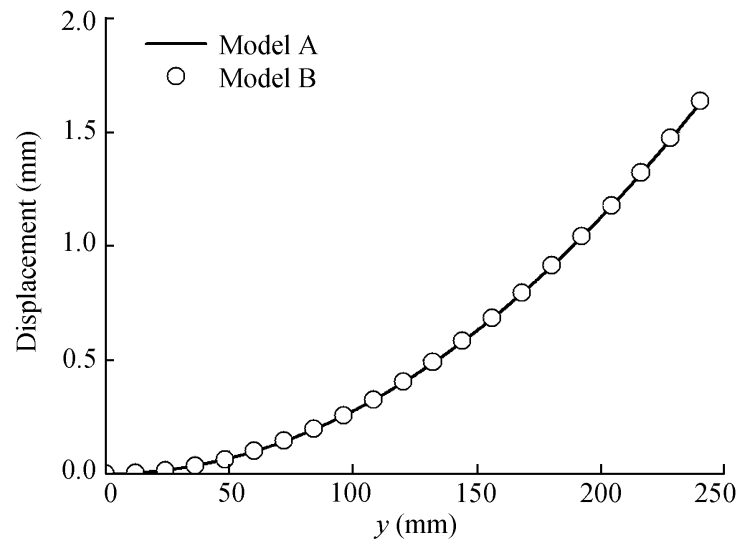

Figure 11 Displacement in the center line of plate middle plane along wingspan.

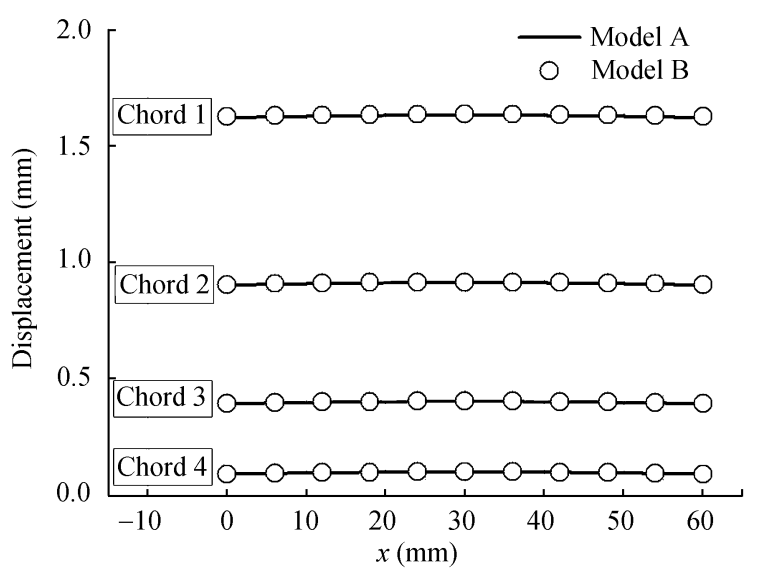

Figure 12 Displacements of plate middle plane along different chords.

ANSYS code. The transfer matrix is given as

$$
\begin{gathered}
c_{x y z}^{\mathrm{E}}=T c_{123}^{\mathrm{E}} R T^{-1} R^{-1}, \\
e_{t}^{x y z}=T e_{t}^{123} T_{3}^{-1},
\end{gathered}
$$

where $c_{x y z}^{\mathrm{E}}$ and $c_{123}^{\mathrm{E}}$ are stiffness matrix respectively in element coordinate and material coordinate, $e^{x y z}$ and $e^{123}$ are piezoelectric stress coefficient matrix respectively in element coordinate and material coordinate. $T$ is the transfer matrix $(6 \times 6)$ for stress (or strain) tensor, $T_{3}$ is orthogonal transfer matrix $(3 \times 3) . R$ is the Reuter transfer matrix from strain tensor to engineering strain.

The numerical results are shown in Figures 13 and 14 which show that the relative error of the maximum displacement for two models is less than $0.3 \%$. And there exists very evident chordwise deformation produced by shear stress. Thus the torsion effect is very helpful for controlling attack angle of wing.

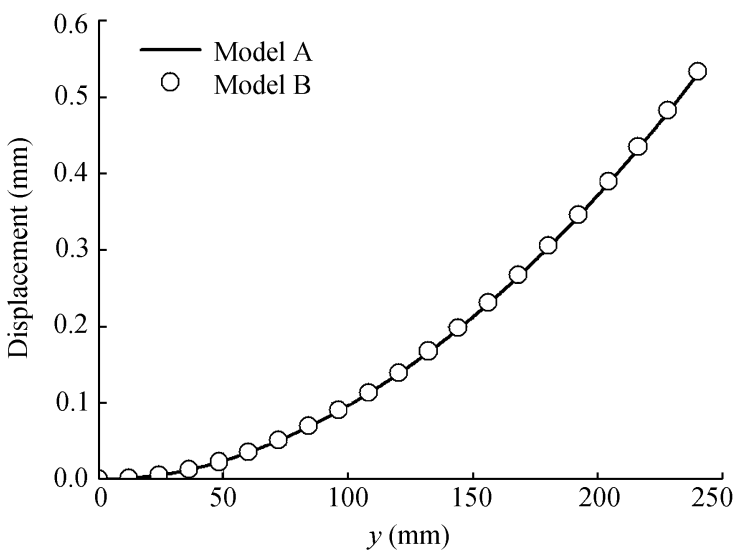

Figure 13 Displacement in the center line of plate middle plane along wingspan.

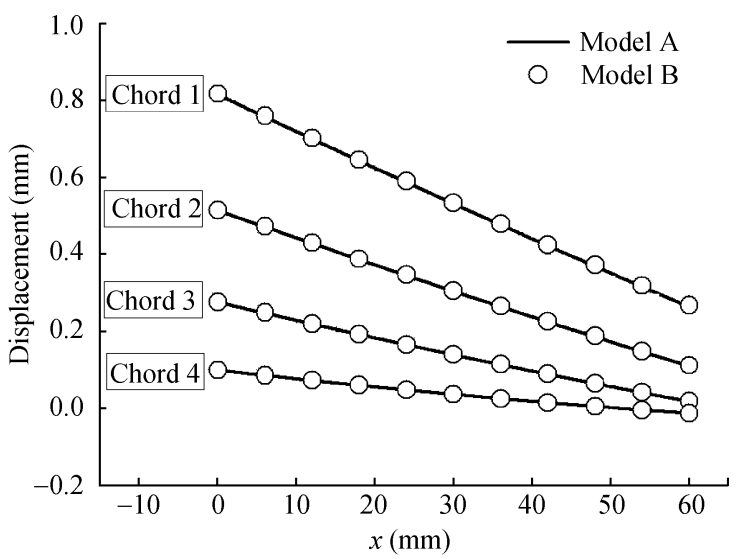

Figure 14 Displacements of plate middle plane along different chords.

\section{Conclusions}

More and more piezoelectric materials and structures have been applied to structure control in aviation and aerospace industry. More efficient and convenient computation method for large complex structure with piezoelectric actuation device is required. The concept of the load simulation method presented in this paper is comprehensible and very suitable for structure strength engineer, thereby is valuable for promoting the application of piezoelectric material and structures to practical engineering. The load simulation method of piezoelectric actuation has the following advantages:

(1) The freedom degrees of finite element model using $2 \mathrm{D}$ plate element but not 3D element are significantly reduced even by $3-6$ orders of magnitude for a practical wing structure.

(2) It is more convenient because one needs not to define the complex in-plane voltage for piezoelectric element in finite element code such as ANSYS. Further- 
more it overcomes the obstacle of difficultly applying voltage on a multi-layers piezoelectric fiber composite for case of sharing multi grids.

(3) Using 2D composite plate element one could easily define material main direction without complex transferring computation between element coordinate

1 Culshaw B. Smart Structures and Materials. Boston: Artech House, 1996, Chap. 1

2 Yousefi-Koma A, Zimcik D G. Applications of smart structures to aircraft for performance enhancement. Canadian Aeronaut Space J, 2003, 49(4): 163-172

3 Niezrecki C, Brei D, Balakrishnan S, et al. Piezoelectric actuation: state of the art. Shock and Vibration Digest, 2001, 33(4): 269280

4 Garcia E. Smart structures and actuators: past, present, and future. Proceedings of SPIE Conference on Smart Structures and Material Systems: Industrial and Commercial Applications of Smart Structures Technologies, Bellingham, WA, vol 4698, 2002. 1-12

5 Schultz M R, Hyer M W. A morphing concept based on unsymmetric composite laminates and piezoceramic MFC actuators, AIAA Paper 2004, 1806

6 Park J S, Kim J H. Material properties of single crystal macro fiber and material coordinate.

The precision of this method is examined and the displacement error is less than $0.3 \%$ for examples in this paper. The method could also be generalized to dynamic response analysis for piezoelectric actuation structure.

composite actuators for active twist rotor blades, AIAA Paper 2005, 2265

7 Williams R B. Nonlinear mechanical and actuation characterization of piezoceramic fiber composites. Doctoral Dissertation. Blacksburg: Virginia Polytechnic Institute and State University, 2004

8 http://www.smart-material.com

9 Carlos E S C, Brown E L. Modeling of high aspect ratio active flexible wings for roll control. AIAA Paper 2002, 1719

10 Sahoo D, Carlos E S C. Roll maneuver control of UCAV wing using anisotropic piezoelectric actuators. AIAA Paper 2002, 1720

11 Bilgen O, Kochersberger K B, Inman D J. A novel aerodynamic vectoring control airfoil via macro-fiber-composite actuators plate airfoil designs. AIAA Paper 2008, 1700

12 Li M, Chen W M, Guan D. Improvement of aircraft rolling power by use of piezoelectric actuators, Chinese J Aeronaut, 2004, 17(2): 8792 